Wu Yang $\bowtie$

Hou $\mathrm{Li}$

Ma Dengqiu

Wei Yongqiao

Luo Lan

https://doi.org/10.21278/TOF.444009419

ISSN 1333-1124

eISSN 1849-1391

\title{
MILLING MACHINE ERROR MODELLING AND ANALYSIS IN THE MACHINING OF CIRCULAR-ARC-TOOTH-TRACE CYLINDRICAL GEARS
}

\begin{abstract}
Summary
This paper is to study errors of a milling machine used for the machining of CircularArc-Tooth-Trace (CATT) cylindrical gears. A topological structure model of the milling machine is established using the lower numbered body array approach. Coordinate transformation matrices between adjacent parts of the milling machine are set up by the D-H homogeneous transformation matrix. Based on the topological structure model and transformation matrices, a comprehensive error model of a milling machine used for the machining of CATT gears is established. According to the error model, the angle errors of the milling point are linear combinations of the angle errors of moving parts, while the displacement errors of the milling point are related to displacement errors, the angle errors of moving parts, the adjustment parameters of machine tools, and the geometric parameters of the gear. Finally, the influence of the errors of the cutter head on the milling point errors in the machining of CATT gears are discussed. The research can provide a theoretical basis for the error analysis, precision design, and the geometric error compensation of a milling machine used for CATT gear machining.
\end{abstract}

Key words: $\quad$ Circular-Arc-Tooth-Trace (CATT) cylindrical gear, milling machine, topological structure model, coordinate transmission matrix, comprehensive error model

\section{Introduction}

Circular Arc Tooth Trace (CATT) transmission is a new type of parallel axle gear wheel transmission. Compared with the traditional spur gear drive and helical gear drive, the CATT drive has better contact and lubrication characteristics and bending strength and it also has lower sensitivity to installation error; in the CATT transmission, there is no axial force because the tooth traces are arcs and the meshing surfaces of the drive is a concave surface and a convex surface [1]. Since the first application of circular arc tooth line to cylindrical gears in 1940s, many scholars have done a great deal of research on the processing method, mathematical model, meshing characteristics and strength of CATT gears. Tseng [2] proposed to machine CATT gears with a double-sided cutter head; the author deduced a mathematical 
model of the gear, discussed the undercutting conditions of the gear, and analysed the contact characteristics of the gear pair. Andrei [3] applied a single-edged cutter head with cutter tilt to the milling of CATT gears and presented a mathematical model of the gear tooth surface. Tseng [4] and Dai Yutang et al. [5] put forward a method of hobbing the CATT gears successively; the authors studied the hobbing mechanism of the gear, realized continuous cutting for the gear, and deduced a mathematical model of the gear. Yi-Cheng $\mathrm{Wu}$ [6] proposed to manufacture CATT gears by using two complementary tooth-shaped cutters. A mathematical model of tooth surface was obtained and the contact characteristics were analysed. Ma Zhenqun [7] put forward a method of producing CATT gears by using a doublesided cutter head with cutter inclination, and realized the CNC modification of the tooth surface of the gear. Zhao Fei [8] studied the CATT gear machined by a rotary cutter head with double edges; the author deduced the equation of the full tooth surface of the CATT gear with transition tooth surface, and proposed a calculation method of the parameter range of the full tooth surface. Using the finite element method, Andrei [9] analysed the stress distribution in the CATT gear machined with a single-edged cutter head with inclination tilt. When compared with a herringbone gear pair with the same parameters, the CATT gear pair exhibited higher bending strength. Fuentes [10] established a model of CATT gears machined by single-edged and double-edged cutter heads and analysed the influence of the matching relationship of cutter head radius on the contact characteristics of the CATT gear pair using the finite element method. Chen Y C [11] modified CATT gears and analysed the contact stress and transmission error of the gear drive by using the finite element method. Sun Zhijun [12] introduced the length coefficient of contact line for the line contact CATT gear pair based on the Hertz contact theory, and established a contact stress calculation model of the gear, which provided a reference for the calculation of the CATT drive contact stress.

However, there is no relevant research on the errors in the machining of CATT gears. The gear machining errors directly affect the contact quality of gears, which affects the contact strength, bending strength, and the fatigue life of gear transmission. V. Simon [13][14] studied the change in the contact stress and the transmission error of spiral bevel gear and hypoid gear drives caused by the change in machine tool adjustment parameters. Tang Jinyuan [15] conducted the contact analysis of spiral bevel gear transmission with errors; the analysis showed that the machine tool motion errors and installation errors had a great influence on the contact quality of the spiral bevel gear tooth surface. A gear machine tool is a multi-body system. Gear machining errors are related to the adjustment parameter errors, motion errors, and component installation errors. Chen Shuhan [16] studied the machining errors of a six-axis NC grinder used for spiral bevel gears; they established a comprehensive error model of the grinder by using the multi-body system theory and homogeneous transformation matrices. Ming Xingzu et al. [17] analysed the geometric error and the thermal error of a face gear NC grinding machine and established a comprehensive mathematical model of the grinding machine error. Zhou Chao [18] studied the relationship between the grinding wheel position errors and the spiral bevel gear tooth surface errors; they also put forward a concept of error-sensitive directions of the spindle eccentricity error and vertical error and a calculation method for determining the error-sensitive direction. Wang Yanzhong et al. [19] analysed the influence of machine tool errors on the surface profile of face gear; in addition, the authors obtained the rule of face gear surface deviation caused by errors of machine tool components and established a mathematical expression of machine tool adjustment parameters.

In this paper, a model of the gear milling machine structure is established first based on the principle of forming the CATT gear. Then, a model of machine tool topological structure is obtained by using the lower numbered body array approach. The machine tool coordinate system is derived using the topological structure model. Subsequently, according to the 
coordinate system, the transformation matrices between adjacent bodies are given by the D-H homogeneous transformation matrix. Finally, the geometric error matrix of the milling point of CATT gear is deduced and the characteristics of geometric errors at the milling point are discussed. An example is given to analyse the influence of motion errors of the cutter head on the geometric errors of milling points.

\section{A model of the CATT gear milling machine topological structure}

In this section, a topological structure model of the CATT gear milling machine is established, which lays a theoretical foundation for the comprehensive error modelling of the gear milling machine. Firstly, according to the principle of forming the CATT gear, a sketch of the gear milling machine model is developed. Then the topological structure of the gear milling machine is described by the Huston lower numbered body array method. A topological structure model and the lower numbered body array of the gear milling machine are obtained.

\subsection{Principle of forming the CATT gear}

The CATT gear is milled by a rotary double-edged cutter head as shown in Fig. 1. The cutter parameters are the same as those of a straight rack cutter. In the figure, $r_{G}$ denotes the gear blank pitch radius, $w_{T}$ denotes the cutter head rotational speed, $w_{G}$ denotes the gear blank rotational speed. The expression $o_{T}-x_{T} y_{T} z_{T}$ denotes the cutter head fixed coordinate system, $o_{W}-x_{W} y_{W} z_{W}$ the gear blank fixed coordinate system, and $o_{G}-x_{G} y_{G} z_{G}$ the moving coordinate system fixed to the gear blank. The rotating cutter head for the machining CATT gear is installed on the spindle of the machine tool and rotates around its own axis $o_{T} z_{T}$. The milling cutter is fixed onto the cutter head and rotates with the cutter head. In the milling process, the workpiece rotates around its own axis $o_{G} z_{G}$, and moves back and forth along the axis $x_{W}$ simultaneously, forming a generating motion with the cutters. The cutters cut concave and convex surfaces of the gear teeth at the same time. The gear model created according to this principle is shown in Fig. 2.

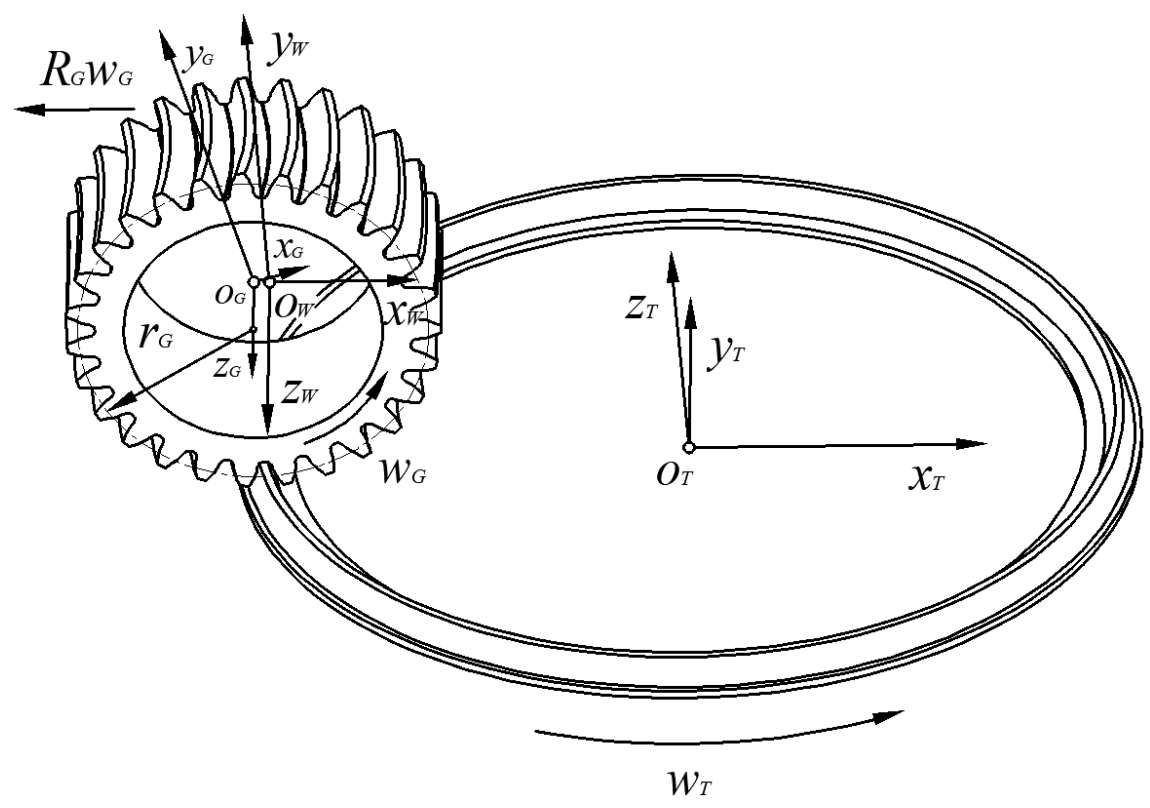

Fig. 1 Principle of forming the CATT gear 
Y. Wu, L. Hou, D. Ma, Y. Wei, L. Luo
Milling Machine Error Modelling and Analysis in the Machining of Circular-Arc-Tooth-Trace Cylindrical Gears

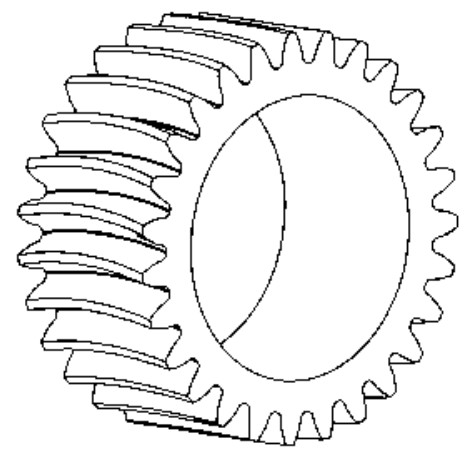

Fig. 2 Three-dimensional model of CATT gear

\subsection{Topological structure of a CATT gear milling machine}

A sketch of the CATT gear milling machine model designed according to the principle of forming the CATT gear is shown in Fig. 3. As one can see in Fig. 3, the CATT gear milling machine system is a typical multi-body system. Therefore, the lower numbered body array method is used to describe the topological structure of the milling machine system.

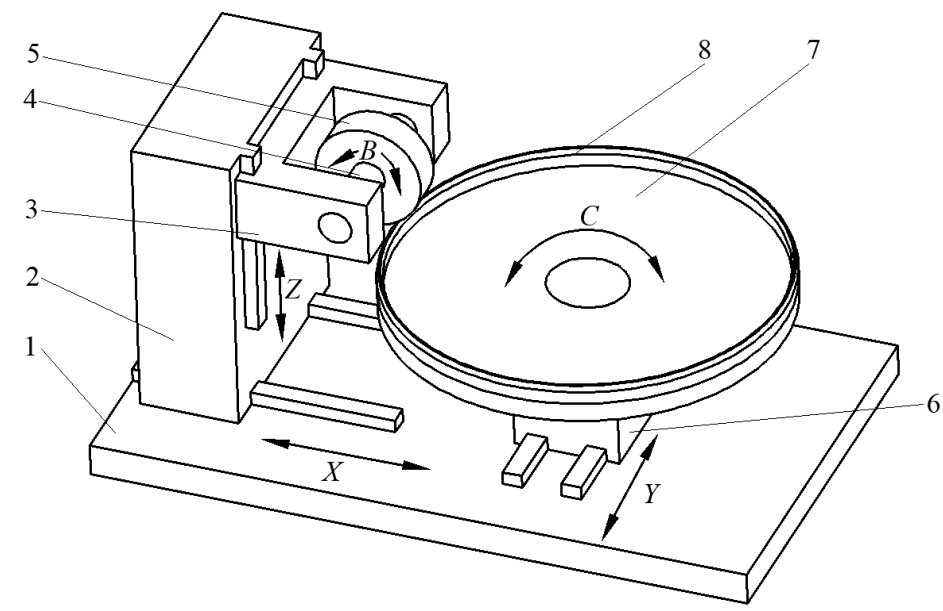

Fig. 3 Sketch of the CATT gear milling machine model

1 bed; $2 X$ axis component; $3 Z$ axis component; $4 B$ axis component; 5 tooth blank; 6 base of cutter head;7 cutter head; 8 cutter

The Huston lower numbered body array method describes the topological structure of a multi-body system by numbering the bodies in the system according to a certain order. This method can effectively describe the relationship between topological structures of a multibody system. The numbering steps are as follows: firstly, set a body in the system as $B_{1}$ and number it as 1; secondly, number each body according to the natural number growth sequence in the direction away from $B_{1}$, from one branch of the system to another, until all the numbering is completed; then, define the body whose number is $i$ as $B_{i}$ [20]. Generally, the system reference coordinate system is set as $B_{0}$ and numbered as 0 .

For an arbitrarily body, $B_{k}$, whose adjacent lower numbered body is $B_{j}$, the lower numbered body array operator $L$ is defined as follows.

$$
L(k)=j
$$

According to the numbering rule of the Huston lower numbered body array method, $L$ operator satisfies

$$
L^{n}(k)=L\left(L^{n-1}(k)\right)
$$


Here, $n$ is a natural number, $n \geq 1$.

A supplementary definition of Equation (2) is

$$
\left\{\begin{array}{c}
L^{0}(k)=k \\
L(0)=0
\end{array}\right.
$$

By applying the Huston lower numbered body array method, the components of gear milling machine are numbered as follows: earth $-B_{0}$, bed $-B_{1}, X$-axis component $-B_{2}, Z$-axis component - $B_{3}, B$-axis component - $B_{4}$, gear blank - $B_{5}$, base of the cutter head - $B_{6}$, cutter head $-B_{7}$. The CATT gear milling system is divided into two branches:

Branch 1: bed $\left(B_{1}\right), X$-axis component $\left(B_{2}\right), Z$-axis component $\left(B_{3}\right), B$-axis component $\left(B_{4}\right)$, and gear blank $\left(B_{5}\right)$;

Branch 2: bed $\left(B_{1}\right)$, base of the cutter head $\left(B_{6}\right)$, and cutter head $\left(B_{7}\right)$.

According to the description of the topological structure of a CATT gear milling machine, we obtain the lower numbered body array of the milling machine, as shown in Table 1, and the schematic diagram of the milling machine topological structure, as shown in Fig.4.

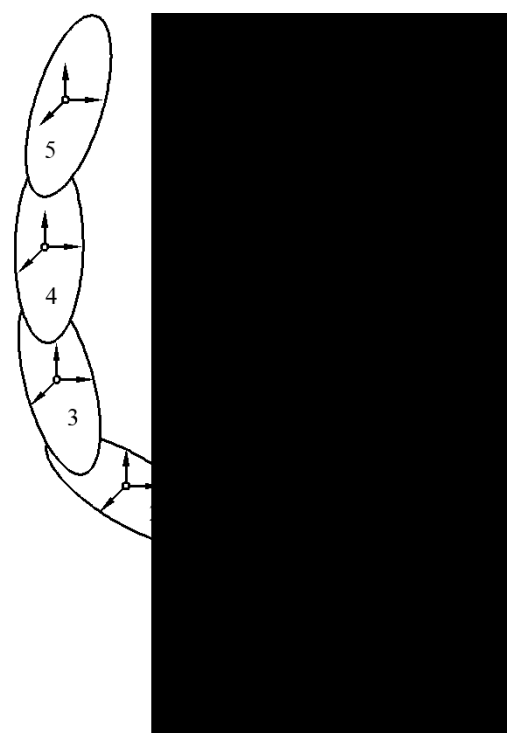

Fig. 4 A schematic diagram of the CATT gear milling machine topological structure

Table 1 Lower numbered body array of the CATT gear milling machine

\begin{tabular}{|l|l|l|l|l|l|l|l|}
\hline Typical Body & 1 & 2 & 3 & 4 & 5 & 6 & 7 \\
\hline$L^{0}(K)$ & 1 & 2 & 3 & 4 & 5 & 6 & 7 \\
\hline$L^{1}(K)$ & 0 & 1 & 2 & 3 & 4 & 1 & 6 \\
\hline$L^{2}(K)$ & 0 & 0 & 1 & 2 & 3 & 0 & 1 \\
\hline$L^{3}(K)$ & 0 & 0 & 0 & 1 & 2 & 0 & 0 \\
\hline$L^{4}(K)$ & 0 & 0 & 0 & 0 & 1 & 0 & 0 \\
\hline$L^{5}(K)$ & 0 & 0 & 0 & 0 & 0 & 0 & 0 \\
\hline
\end{tabular}

\section{Comprehensive error modelling for CATT gear milling machine}

In this section, a comprehensive error model of the CATT gear milling machine is established based on the topological structure model of the gear milling machine. Firstly, the coordinate system of the CATT gear milling machine system is built according to the topological structure model of the gear milling machine, and the motion errors of each part in 
the machine tool are analysed. Then, based on the hypothesis of small error, the coordinate transformation matrices between adjacent parts of the machine tool are established by using the D-H homogeneous transformation matrix. Finally, based on the coordinate transformation matrices between adjacent parts, a comprehensive error model of the machine tool is established. The relationship between errors of the milling point and the motion errors of each part in the milling machine is analysed.

\subsection{Error analysis of the CATT gear milling machine}

According to the model of the milling machine structure shown in Fig. 3 and the description of the milling machine topology in Table 1 and Fig. 4, the coordinate system of the CATT gear milling machine system is established, as shown in Fig. 5, where $o_{i}-x_{i} y_{i} z_{i}$ is the coordinate system of $B_{i}\left(i=1,2,3,4^{\prime}, 4,6,6^{\prime}, 7\right), H_{t}$ is the horizontal cutter location, $V_{t}$ is the vertical cutter location, $H_{w}$ is the horizontal wheel position, $V_{w}$ is the vertical wheel position, and $x$ is the displacement along $x_{1}$ during the processing of gears.

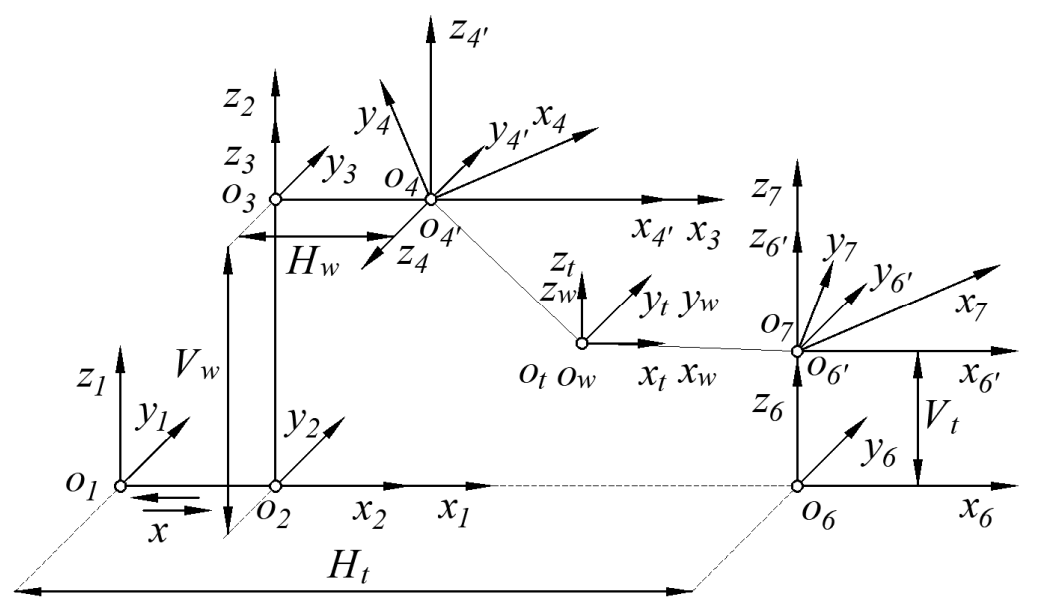

Fig. 5 Coordinate system of the CATT gear milling machine system

In order to facilitate calculation, the D-H homogeneous transformation matrix is used to describe the relative position between adjacent components. When errors exist, the transformation matrix of the body $B_{v}$ to its adjacent low-order body $B_{s}$ is as follows [21]:

$$
M_{s v}={ }^{i} M_{s v}^{p} E_{s v}^{p i} M_{s v}^{s} E_{s v}^{s}
$$

Here, ${ }^{i} M_{s v}^{p}$ is the position transformation matrix from $B_{v}$ to $B_{s}$ under ideal condition, ${ }^{i} M_{s v}^{s}$ is the displacement transformation matrix from $B_{v}$ to $B_{s}$ under ideal condition, $E_{s v}^{p}$ is the position error matrix from $B_{v}$ to $B_{s}, E_{s v}^{s}$ is the displacement error matrix from $B_{v}$ to $B_{s}$.

Considering errors, the transformation matrix of the body $B_{k}$ to its arbitrary low-order body $B_{o}$ in the same branch is as follows [21]:

$$
M_{o k}=\prod_{t=0}^{u}\left[{ }^{i} M_{s v}^{p} E_{s v}^{p i} M_{s v}^{s} E_{s v}^{s}\right]
$$

Here, $s=L^{(t+1)}(k), v=L^{t}(k), u$ is the number of bodies between $B_{k}$ and $B_{o}$ in the same branch.

Because of assembly errors, manufacturing errors, and the wear of the milling machine components, there are always three angular errors $\{\alpha, \beta, \gamma\}$ around the coordinate axes and 
three translation errors $\{\Delta X, \Delta Y, \Delta Z\}$ along the coordinate axes, irrespective of whether the body $B_{v}$ moves or rotates relative to its low-order body $B_{s}$. Each of these errors is small. According to the hypothesis of small error, the position error matrix can be obtained using the D-H homogeneous transformation matrix [22]:

$$
E=\left[\begin{array}{cccc}
1 & -\gamma & \beta & \Delta X \\
\gamma & 1 & -\alpha & \Delta Y \\
-\beta & \alpha & 1 & \Delta Z \\
0 & 0 & 0 & 1
\end{array}\right]
$$

In the actual machining of CATT gears, the milling machine system gradually transitions from a non-stationary state to a stationary state, and the machine temperature rises first and then stabilizes near a certain value. The motion errors between adjacent bodies are functions of the temperature. By analysing the structure of the milling machine, it can be seen that the CATT gear milling machine contains 33 geometric and thermal error elements, as shown below.

Motion errors of $B_{2}$ : linear displacement error, $\Delta X(x, t)$, straightness error along $y_{2}, \Delta Y(x, t)=\int_{0}^{x} \gamma(x, t) d x$, straightness error along $z_{2}, \Delta Z(x, t)=-\int_{0}^{x} \beta(x, t) d x$; roll error $\alpha(x, t)$, pitch error $\beta(x, t)$, and yaw error $\gamma(x, t)$.

Motion errors of $B_{6}$ : linear displacement error, $\Delta Y(y, t)$, straightness error along $x_{6}, \Delta X(\mathrm{y}, t)=-\int_{0}^{y} \gamma(y, t) d y$, straightness error along $z_{6}, \Delta Z(\mathrm{y}, t)=\int_{0}^{y} \alpha(y, t) d y$; roll error $\beta(y, t)$, pitch error $\alpha(y, t)$, and yaw error $\chi(y, t)$.

Motion errors of $B_{3}$ : linear displacement error, $\Delta Z(z, t)$, straightness error along $x_{3}, \Delta X(z, t)=\int_{0}^{z} \beta(z, t) d z$, straightness error along $y_{3}, \Delta Y(z, t)=-\int_{0}^{z} \alpha(z, t) d z$; roll error $\gamma(z, t)$, pitch error $\beta(z, t)$, and yaw error $\alpha(z, t)$.

Motion errors of $B_{4}$ : linear displacement errors, $\Delta X(\psi, t), \Delta Y(\psi, t)$, and $\Delta Z(\psi, t)$ along $x_{4}, y_{4}$, and $z_{4}$, respectively; angle errors $\alpha(\psi, t), \beta(\psi, t)$, and $\gamma(\psi, t)$ around $x_{4}$, $y_{4}$, and $z_{4}$, respectively.

Motion errors of $B_{7}$ : linear displacement errors $\Delta X\left(\theta_{z}, t\right), \Delta Y\left(\theta_{z}, t\right)$, and $\Delta Z\left(\theta_{z}, t\right)$ along $x_{\sigma^{\prime}}, y_{\sigma^{\prime}}$, and $z_{6}$, respectively; angle errors $\alpha\left(\theta_{z}, t\right), \beta\left(\theta_{z}, t\right)$, and $\gamma\left(\theta_{z}, t\right)$ around $x_{6^{\prime}}$, $y_{6}$, and $z_{6},{ }^{\prime}$, respectively.

Due to the manufacturing and assembly errors, there are also vertical errors between $X, Y$, and $Z$ axes, $\varepsilon_{x y}, \varepsilon_{y z}$, and $\varepsilon_{z x}$.

\subsection{Coordinate transformation matrix between adjacent bodies}

The transformation matrix between adjacent bodies can be established based on the analysis presented above.

When there is no error, the transformation matrices between components are as follows:

Coordinate transformation matrix from $B_{2}$ to $B_{1}$ : 
Y. Wu, L. Hou, D. Ma,

Y. Wei, L. Luo
Milling Machine Error Modelling and Analysis in the Machining of Circular-Arc-Tooth-Trace Cylindrical Gears

$$
{ }^{i} A_{12}=\left[\begin{array}{llll}
1 & 0 & 0 & x \\
0 & 1 & 0 & 0 \\
0 & 0 & 1 & 0 \\
0 & 0 & 0 & 1
\end{array}\right]
$$

Coordinate transformation matrix from $B_{3}$ to $B_{2}$ :

$$
{ }^{i} A_{23}=\left[\begin{array}{cccc}
1 & 0 & 0 & 0 \\
0 & 1 & 0 & 0 \\
0 & 0 & 1 & V_{w} \\
0 & 0 & 0 & 1
\end{array}\right]
$$

Coordinate transformation matrix from $B_{4}$ to $B_{3}$ :

$$
\begin{aligned}
A_{34^{\prime}} & =\left[\begin{array}{cccc}
1 & 0 & 0 & H_{w} \\
0 & 0 & -1 & 0 \\
0 & 1 & 0 & 0 \\
0 & 0 & 0 & 1
\end{array}\right] \\
{ }^{i} A_{4^{\prime} 4} & =\left[\begin{array}{cccc}
\cos \psi & -\sin \psi & 0 & 0 \\
\sin \psi & \cos \psi & 0 & 0 \\
0 & 0 & 1 & 0 \\
0 & 0 & 0 & 1
\end{array}\right]
\end{aligned}
$$

Coordinate transformation matrix from $B_{6}$ to $B_{1}$ :

$$
{ }^{i} A_{16}=\left[\begin{array}{cccc}
1 & 0 & 0 & H_{t} \\
0 & 1 & 0 & 0 \\
0 & 0 & 1 & 0 \\
0 & 0 & 0 & 1
\end{array}\right]
$$

Coordinate transformation matrix from $B_{7}$ to $B_{6}$ :

$$
\begin{aligned}
A_{66^{\prime}} & =\left[\begin{array}{llll}
1 & 0 & 0 & 0 \\
0 & 1 & 0 & 0 \\
0 & 0 & 1 & V_{t} \\
0 & 0 & 0 & 1
\end{array}\right] \\
{ }^{i} A_{6^{\prime} 7} & =\left[\begin{array}{llll}
1 & 0 & 0 & 0 \\
0 & 1 & 0 & 0 \\
0 & 0 & 1 & 0 \\
0 & 0 & 0 & 1
\end{array}\right]
\end{aligned}
$$

When errors exist, the transformation matrices between components are as follows:

Coordinate transformation matrix from $B_{2}$ to $B_{1}$ : 
Milling Machine Error Modelling and Analysis in the Machining of Circular-Arc-Tooth-Trace Cylindrical Gears
Y. Wu, L. Hou, D. Ma, Y. Wei, L. Luo

$$
\begin{aligned}
A_{12} & =\left[\begin{array}{cccc}
1 & -\gamma(x, t) & \beta(x, t) & x+\Delta X(x, t) \\
\gamma(x, t) & 1 & -\alpha(x, t) & \Delta Y(x, t) \\
-\beta(x, t) & \alpha(x, t) & 1 & \Delta Z(x, t) \\
0 & 0 & 0 & 1
\end{array}\right] \\
B_{12} & =\left[\begin{array}{cccc}
1 & 0 & \varepsilon_{z x} & 0 \\
0 & 1 & -\varepsilon_{y z} & 0 \\
-\varepsilon_{z x} & \varepsilon_{y z} & 1 & 0 \\
0 & 0 & 0 & 1
\end{array}\right]
\end{aligned}
$$

Coordinate transformation matrix from $B_{3}$ to $B_{2}$ :

$$
A_{23}=\left[\begin{array}{cccc}
1 & -\gamma(z, t) & \beta(z, t) & \Delta X(z, t) \\
\gamma(z, t) & 1 & -\alpha(z, t) & \Delta Y(z, t) \\
-\beta(z, t) & \alpha(z, t) & 1 & V_{w}+\Delta Z(z, t) \\
0 & 0 & 0 & 1
\end{array}\right]
$$

Coordinate transformation matrix from $B_{4}$ to $B_{3}$ :

$$
A_{4^{\prime} 4}=\left[\begin{array}{cccc}
\cos (\psi+\gamma(\psi, t)) & -\sin (\psi+\gamma(\psi, t)) & \beta(\psi, t) & \Delta X(\psi, t) \\
\sin (\psi+\gamma(\psi, t)) & \cos (\psi+\gamma(\psi, t)) & -\alpha(\psi, t) & \Delta Y(\psi, t) \\
\alpha(\psi, t) \sin (\psi+\gamma(\psi, t)) & \beta(\psi, t) \sin (\psi+\gamma(\psi, t)) & & \Delta Z(\psi, t) \\
-\beta(\psi, t) \cos (\psi+\gamma(\psi, t)) & +\alpha(\psi, t) \cos (\psi+\gamma(\psi, t)) & 1 & \\
0 & 0 & 0 & 1
\end{array}\right]
$$

Coordinate transformation matrix from $B_{6}$ to $B_{1}$ :

$$
\begin{aligned}
A_{16} & =\left[\begin{array}{cccc}
1 & -\gamma(y, t) & \beta(y, t) & H_{t}+\Delta X(y, t) \\
\gamma(y, t) & 1 & -\alpha(y, t) & \Delta Y(y, t) \\
-\beta(y, t) & \alpha(y, t) & 1 & \Delta Z(y, t) \\
0 & 0 & 0 & 1
\end{array}\right] \\
B_{16} & =\left[\begin{array}{cccc}
1 & -\varepsilon_{x y} & 0 & 0 \\
\varepsilon_{x y} & 1 & 0 & 0 \\
0 & 0 & 1 & 0 \\
0 & 0 & 0 & 1
\end{array}\right]
\end{aligned}
$$

Coordinate transformation matrix from $B_{7}$ to $B_{6}$ :

$$
A_{6^{\prime} 7}=\left[\begin{array}{cccc}
1 & -\gamma\left(\theta_{z}, t\right) & \beta\left(\theta_{z}, t\right) & \Delta X\left(\theta_{z}, t\right) \\
\gamma\left(\theta_{z}, t\right) & 1 & -\alpha\left(\theta_{z}, t\right) & \Delta Y\left(\theta_{z}, t\right) \\
-\beta\left(\theta_{z}, t\right) & \alpha\left(\theta_{z}, t\right) & 1 & \Delta Z\left(\theta_{z}, t\right) \\
0 & 0 & 0 & 1
\end{array}\right]
$$




\subsection{Establishment of a comprehensive error model for a CATT gear milling machine}

Suppose that a milling point on the CATT gear tool surface is $\mathrm{P}_{t}$, the coordinate system at $\mathrm{P}_{\mathrm{t}}$ is $o_{t}-x_{t} y_{t} z_{t}$, the corresponding milling point on the workpiece surface is $\mathrm{P}_{\mathrm{w}}$, and the coordinate system at $\mathrm{Pw}$ is $o_{w}-x_{w} y_{w} z_{w}$, as shown in Fig. 5. When there is no error, $o_{t}-x_{t} y_{t} z_{t}$ and $o_{w}-x_{w} y_{w} z_{w}$ coincide, and there is no machining error for the tooth surface. From Fig. 4 and Equations (4) and (5), the transformation matrix from the cutter head coordinate system to the gear blank coordinate system can be deduced as follows:

$$
\begin{aligned}
& { }^{i} M_{47}=\left({ }^{i} M_{14}\right){ }^{-1}{ }^{i} M_{17} \\
& { }^{i} M_{14}={ }^{i} A_{12}{ }^{i} A_{23} A_{34^{\prime}}{ }^{i} A_{4^{\prime} 4} \\
& { }^{i} M_{17}={ }^{i} A_{16} A_{66^{\prime}}{ }^{i} A_{6^{\prime} 7}
\end{aligned}
$$

When there are errors, $o_{t}-x_{t} y_{t} z_{t}$ and $o_{w}-x_{w} y_{w} z_{w}$ will be separated, resulting in milling errors of the CATT gear tooth surface. From Equation (4) and (5), the transformation matrix from the cutter head coordinate system to the gear blank coordinate system can be derived as follows:

$$
\begin{aligned}
& M_{47}=\left(M_{14}\right)^{-1} M_{17} \\
& M_{14}=B_{12} A_{12} A_{23} A_{34^{\prime}} A_{4^{\prime} 4} \\
& M_{17}=B_{16} A_{16} A_{66^{\prime}} A_{6^{\prime} 7}
\end{aligned}
$$

According to Equation (4), $M_{47}$ can be expressed as below:

$$
M_{47}={ }^{i} M_{47} E_{t}
$$

Here: $E_{t}$ is the position error matrix of the cutter head.

According to Equation (27), the position error matrix of the cutter head is:

$$
E_{t}=\left({ }^{i} M_{47}\right)^{-1} M_{47}
$$

Then the position error matrix for different milling points can be expressed as:

$$
E=r_{2} E_{t} r_{1}
$$

Here, $r_{1}$ is the transformation matrix from the actual milling point coordinate system to the actual cutter head coordinate system, and $r_{2}$ is the transformation matrix from the theoretical cutter head coordinate system to the theoretical milling point coordinate system. 


$$
\begin{aligned}
& r_{1}=\left[\begin{array}{cccc}
1 & 0 & 0 & -\left(R_{t} \mp \frac{\pi}{4} m \pm u \sin \alpha\right) \cos \theta \\
0 & 1 & 0 & -\left(R_{t} \mp \frac{\pi}{4} m \pm u \sin \alpha\right) \sin \theta \\
0 & 0 & 1 & u \cos \alpha \\
0 & 0 & 0 & 1
\end{array}\right] \\
& r_{2}=\left[\begin{array}{lllc}
1 & 0 & 0 & \left(R_{t} \mp \frac{\pi}{4} m \pm u \sin \alpha\right) \cos \theta \\
0 & 1 & 0 & \left(R_{t} \mp \frac{\pi}{4} m \pm u \sin \alpha\right) \sin \theta \\
0 & 0 & 1 & -u \cos \alpha \\
0 & 0 & 0 & 1
\end{array}\right]
\end{aligned}
$$

Here, ' \pm ' denotes the inside and outside edges of the cutter, respectively, and the upper part of the symbol is taken for the inner edge; $\theta$ is the phase of the milling point, $-\frac{1}{2} \arcsin \left(\frac{b}{R_{t}}\right) \leq \theta \leq \frac{1}{2} \arcsin \left(\frac{b}{R_{t}}\right), b$ is the tooth width, $R_{t}$ is the radius of the cutter head.

According to the hypothesis of small error, E can be given as follows:

$$
E=\left[\begin{array}{cccc}
1 & -\phi_{z} & \phi_{y} & \Delta \delta_{x} \\
\phi_{z} & 1 & -\phi_{x} & \Delta \delta_{y} \\
-\phi_{y} & \phi_{x} & 1 & \Delta \delta_{z} \\
0 & 0 & 0 & 1
\end{array}\right]
$$

Insert all the errors into Equation (29) and neglect the error terms of quadratic and over; each error in Equation (32) can be obtained by considering the influence of all motion errors of components in the CATT gear milling machine.

$$
\begin{aligned}
\Delta \phi_{x} & =\alpha(y, t)-\alpha(x, t)-\alpha(z, t)-\alpha(\psi, t)+\alpha\left(\theta_{z}, t\right)-\varepsilon_{y z} \\
\Delta \phi_{y} & =\beta(y, t)-\beta(x, t)-\beta(z, t)+\beta\left(\theta_{z}, t\right)+\gamma(\psi, t)-\varepsilon_{z x} \\
\Delta \phi_{z} & =\beta(\psi, t)+\gamma(x, t)-\gamma(y, t)+\gamma(z, t)-\gamma\left(\theta_{z}, t\right)-\varepsilon_{x y} \\
\Delta \delta_{x} & =\Delta X(y, t)-\Delta X(x, t)-\Delta X(z, t)-\Delta X(\psi, t)+\Delta X\left(\theta_{z}, t\right)+(\beta(z, t)-\gamma(\psi, t)) V_{w} \\
& -\left(\beta(x, t)-\beta(y, t)+\beta(z, t)-\gamma(\psi, t)+\varepsilon_{z x}\right) V_{t} \\
& -\left(\beta(x, t)-\beta(y, t)+\beta(z, t)-\beta\left(\theta_{z}, t\right)-\gamma(\psi, t)+\varepsilon_{z x}\right) u \cos \alpha \\
& -\left(\beta(\psi, t)+\gamma(x, t)-\gamma(y, t)+\gamma(z, t)-\gamma\left(\theta_{z}, t\right)-\varepsilon_{x y}\right)\left(R_{t} \mp \frac{\pi}{4} m \pm u \sin \alpha\right) \sin \theta
\end{aligned}
$$




$$
\begin{aligned}
\Delta \delta_{y} & =\Delta Y(y, t)-\Delta Y(x, t)-\Delta Y(z, t)+\Delta Y(\psi, t)+\Delta Y\left(\theta_{z}, t\right) \\
& +(\beta(\psi, t)+\gamma(x, t)+\gamma(z, t)) x-(\alpha(z, t)+\alpha(\psi, \mathrm{t})) H_{v} \\
& -\left(\beta(\psi, t)+\gamma(x, t)+\gamma(z, t)-\varepsilon_{x y}\right) x_{o 6}^{(1)}+\beta(\psi, \mathrm{t}) H_{w} \\
& +\left(\alpha(x, t)-\alpha(y, t)+\alpha(z, t)+\alpha(\psi, t)+\varepsilon_{y z}\right) V_{t} \\
& +\left(\alpha(x, t)-\alpha(y, t)+\alpha(z, t)+\alpha(\psi, \mathrm{t})-\alpha\left(\theta_{z}, t\right)+\varepsilon_{y z}\right) u \cos \alpha \\
& +\left(\beta(\psi, t)+\gamma(x, t)-\gamma(y, t)+\gamma(z, t)-\gamma\left(\theta_{z}, t\right)-\varepsilon_{x y}\right)\left(R_{t} \mp \frac{\pi}{4} m \pm u \sin \alpha\right) \cos \theta \\
\Delta \delta_{z} & =\Delta Z(y, t)-\Delta Z(x, t)-\Delta Z(z, t)-\Delta Z(\psi, t)+\Delta Z\left(\theta_{z}, t\right)+\gamma(\psi, t) H_{w} \\
& -(\beta(x, t)+\beta(\mathrm{z}, t)-\gamma(\psi, t)) x+\left(\beta(x, t)+\beta(\mathrm{z}, t)-\gamma(\psi, t)+\varepsilon_{z x}\right) H_{t} \\
& +\left(\alpha(x, t)-\alpha(\mathrm{y}, t)+\alpha(\mathrm{z}, t)+\alpha(\psi, t)-\alpha\left(\theta_{z}, t\right)+\varepsilon_{y z}\right)\left(R_{t} \mp \frac{\pi}{4} m \pm u \sin \alpha\right) \sin \theta \\
& -\left(\beta(x, t)-\beta(\mathrm{y}, t)+\beta(\mathrm{z}, t)-\beta\left(\theta_{z}, t\right)-\gamma(\psi, t)+\varepsilon_{z x}\right)\left(R_{t} \mp \frac{\pi}{4} m \pm u \sin \alpha\right) \cos \alpha
\end{aligned}
$$

From Equations (33)-(38), one can see that there is no coupling term between the motion errors of parts and the errors of the milling point. The angle errors of the milling point are a linear combination of the angle errors of the moving parts. When the phase of the milling point is a constant, the displacement errors of the milling point are linear groups of the displacement errors of the moving parts and the displacement errors caused by the angle errors. The displacement errors of milling points are related to the displacement errors and angle errors of the moving parts, adjustment parameters and motion parameters of machine tools, and geometric parameters of machined gears.

\section{Example}

In this section, the processing of the CATT gear concave surface is taken as an example to analyse the influence of component motion errors on the errors of milling points. Due to the limited space, only the influence of the position errors of $B_{7}$ (cutter head) on the errors of the milling point is discussed. The number of gear teeth is 49 , the modulus is $4 \mathrm{~mm}$, and the tooth width is $100 \mathrm{~mm}$. The machine tool adjustment parameters are given in Table 2 .

The variation range of angle errors of $B_{7}$ is set as $-10 \times 10^{-5} \mathrm{rad} \sim 10 \times 10^{-5} \mathrm{rad}$, and the variation range of displacement errors is $-10 \mu \mathrm{m} \sim 10 \mu \mathrm{m}$. By inserting the motion errors of $B_{7}$ into Equation (33)-(38), the influence of the motion errors of $B_{7}$ on the position error of milling points can be obtained.

Table 2 Adjustment parameters of the CATT gear milling machine

\begin{tabular}{|c|c|}
\hline Adjustment parameter & Value \\
\hline Tooth angle of cutter head $\alpha_{c}\left({ }^{\circ}\right)$ & 22.5 \\
\hline Cutter head radius $R_{t}(\mathrm{~mm})$ & 300 \\
\hline$H_{t}(\mathrm{~mm})$ & 600 \\
\hline$V_{t}(\mathrm{~mm})$ & 200 \\
\hline$H_{w}(\mathrm{~mm})$ & 200 \\
\hline$V_{w}(\mathrm{~mm})$ & 298 \\
\hline
\end{tabular}




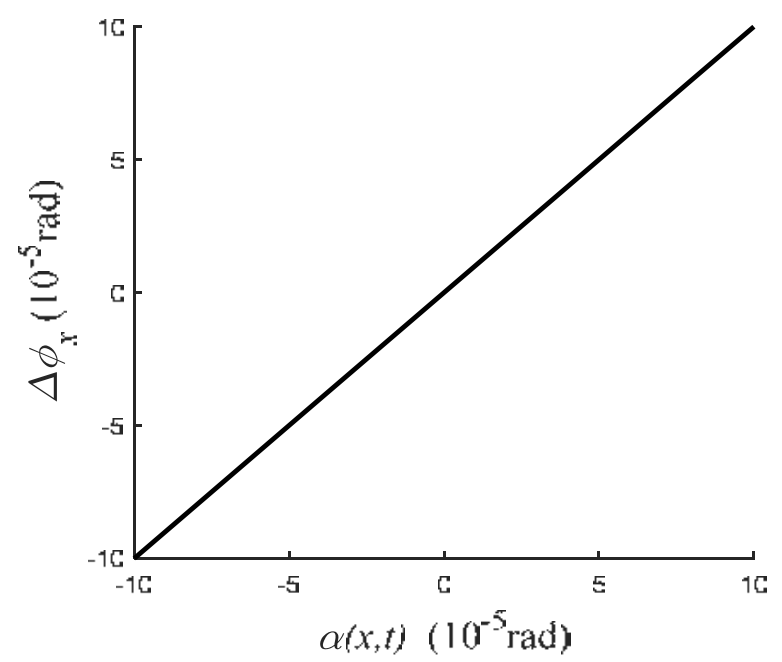

Fig. 6 The relationship between the angle error around the $x$-axis of the milling point and the angle error around the $x$-axis of $B_{7}$

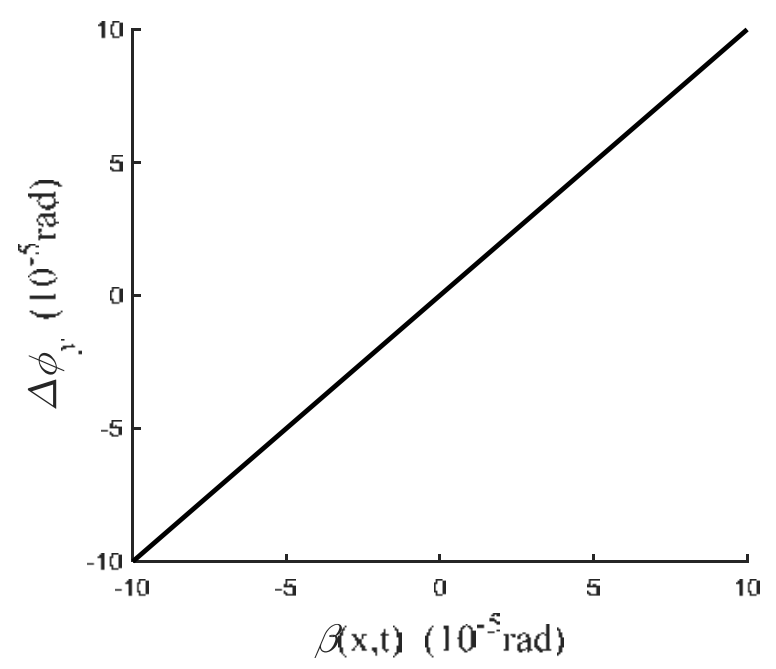

Fig. 7 The relationship between the angle error around the $y$-axis of the milling point and the angle error around the $y$-axis of $B_{7}$

Figures 6 and 7 show the relationship between the angular errors of the milling points and the motion errors of $B_{7}$. It can be seen from the figures that the angular errors of the milling points are only related to the angular errors of the corresponding axis of $B_{7}$. From Fig. 6 , the angle error around the $x$-axis of the milling point is proportional to the angle error around the $x$-axis of $B_{7}$, which varies from $-10 \times 10^{-5} \mathrm{rad}$ to $+10 \times 10^{-5} \mathrm{rad}$. This error will mainly lead to the rotation error around the $x$-axis of the tooth surface and to the overcutting (or undercutting) of the tooth surface. From Fig. 7 one can see that the angle error around the $y$ axis of the milling point is proportional to that around the $y$-axis of $B_{7}$, and its range is also from $-10 \times 10^{-5} \mathrm{rad}$ to $+10 \times 10^{-5} \mathrm{rad}$. This error will mainly cause the pressure angle error of the tooth surface. 
Y. Wu, L. Hou, D. Ma, Y. Wei, L. Luo
Milling Machine Error Modelling and Analysis in the Machining of Circular-Arc-Tooth-Trace

Cylindrical Gears

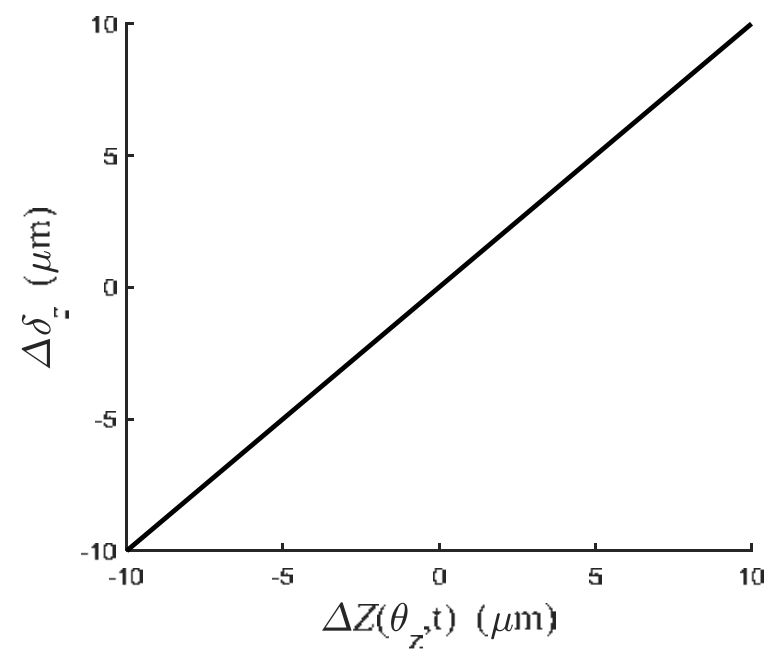

Fig.8 Relation between the displacement error along the $z$-axis of the milling point and the displacement error along the $z$-axis of $B_{7}$

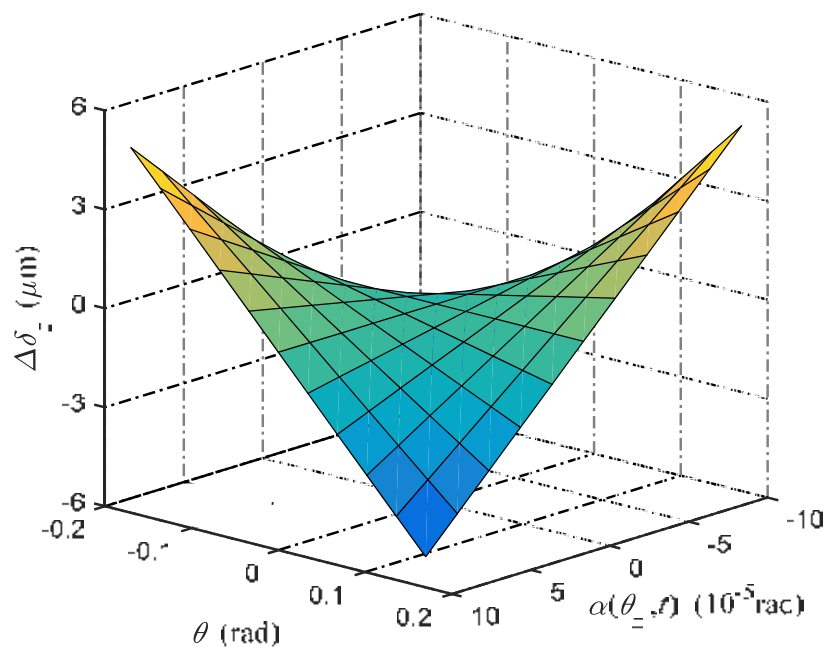

Fig.9 Relation between the displacement error along the $z$-axis of the milling point and the angle error around the $x$-axis of $B_{7}$

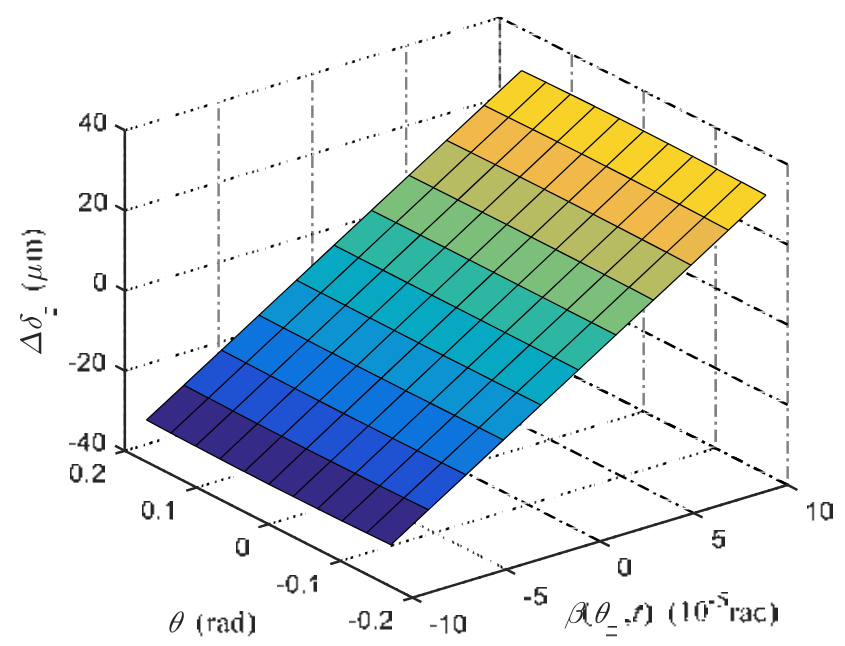

Fig. 10 Relation between the displacement error along the $z$-axis of the milling point and the angle error around the $y$-axis of $B_{7}$ 
Figures 8-10 show the relationship between the displacement error along the z-axis of the milling point and the motion errors of $B_{7}$. According to the figures, the displacement error along the $z$-axis of the milling point is related to the angle errors and displacement errors of $B_{7}$, and the phase of the milling point. The displacement error along the $z$-axis of the milling point will lead to the offset of the tooth surface along the $z$-axis resulting in the asymmetry of the tooth surface with respect to the middle section of the gear. Figure 8 shows that the displacement error along the $z$-axis of the milling point is proportional to the displacement error along the $z$-axis of $B_{7}$ when only the displacement error along the $z$-axis of $B_{7}$ is taken into account, and the range is from $-10 \mu \mathrm{m}$ to $+10 \mu \mathrm{m}$. Figure 9 shows that the displacement error along the z-axis of the milling point is a non-linear function of the phase of the milling point and the angle error around the $x$-axis of $B_{7}$ when only the angle error around the $x$-axis of $B_{7}$ is considered. When the angle error around the $x$-axis of $B_{7}$ is positive, the $z$-axis displacement error of the milling point decreases gradually with an increase in the milling phase. When the angle error around the $x$-axis of $B_{7}$ is negative, the $z$-axis displacement error of the milling point increases gradually with an increase in the milling phase. The $z$-axis displacement error of the milling point tends to zero when the milling point is located in the middle section of the gear, and it reaches its maximum or minimum at the end of the tooth surface. The range of variation is $-5.07 \mu \mathrm{m}$ to $5.07 \mu \mathrm{m}$. Figure 10 shows that the $z$-axis displacement error of the milling point is slightly affected by the phase of the milling point when only the $y$-axis angle error of $B_{7}$ is taken into account. When the phase of the milling point is constant, the $z$-axis displacement error of the milling point is proportional to the $y$ axis angle error of $B_{7}$, and its variation range is $-29.57 \mu \mathrm{m}$ to $29.57 \mu \mathrm{m}$. Comparing Fig. 8, Fig.9 and Fig.10, one can see that the angle error around the $y$-axis of $B_{7}$ has the greatest influence on the displacement error along the $z$-axis of the milling point, and that it is not affected by the milling point phase; the influence of the displacement error along the $z$-axis takes the second place, while the influence of the angle error around the $x$-axis of $B_{7}$ is the smallest.

By introducing the motion errors of $B_{7}$ into Equations (36) and (37), the influence of the motion errors of $B_{7}$ on the displacement errors along the $x$-axis and $y$-axis of the milling point can be obtained. However, since the relationship between the displacement errors along the $x$ and $y$-axes of the milling point and the motion errors of $B_{7}$ is similar to that between the displacement errors along the $z$-axis of the milling point and the motion errors of $B_{7}$; the relationships between the displacement errors along the $x$ - and $y$-axes of the milling point and the motion errors of $B_{7}$ are not discussed here.

\section{Conclusion}

1. According to the model of the CATT gear milling machine, a model of the CATT gear milling machine topological structure is established by the Huston lower numbered body array method; based on that model, the coordinate system of the CATT gear milling machine is established.

2. The error elements of the CATT gear milling machine are analysed based on the motion mode and spatial allocation of components. According to the hypothesis of small error, the coordinate transformation matrices of adjacent components are set up using the D-H homogeneous transformation matrix, and a comprehensive error model of the CATT gear milling machine is deduced. The relationship between the position error of the milling point 
and the motion error of each component is discussed. The comprehensive error model of the CATT gear milling machine provides a theoretical basis for the error analysis, accuracy allocation, and error compensation of the CATT gear milling machine system.

3. Based on the comprehensive error model of the CATT gear milling machine, the influence of motion errors of $B_{7}$ on the position errors of milling points is analysed as an example, and the influence of motion errors of components and the phase of milling points on the position error of milling points is discussed. In addition, the effect of position errors of the milling point on the CATT gear tooth surface morphology is also discussed; this makes the mechanism of tooth surface error clear.

\section{Funding}

This research is supported by the National Natural Science Foundation of China (Grant No. 51375320 and No. 5187052280), the Natural Science Foundation of Gansu Province (Grant No. 18JR3RA140), the Natural Science Foundation of Education Department of Guizhou Province(Grant No. QianjiaoheKYzi[2018]319Hao).

\section{REFERENCES}

[1] Zhao Fei. Research on Forming Theory and Meshing Characteristics of Variable-Hyperbolic -CircularArc-Tooth Cylindrical Gear [D]. Chengdu: Sichuan University, 2018.

[2] Tseng R T, Tsay C B. Mathematical model and undercutting of cylindrical gears with curvilinear shaped teeth[J]. Mechanism \& Machine Theory, 2001, 36(11-12):1189-1202. https://doi.org/10.1016/s0094114x(01)00049-0

[3] Andrei L, Andrei G, Epureanu A, et al. Numerical simulation and generation of curved face width gears[J]. International Journal of Machine Tools \& Manufacture, 2002, 42(1):1-6. https://doi.org/10.1016/s0890-6955(01)00101-8

[4] Tseng J T, Tsay C B. Mathematical Model and Surface Deviation of Cylindrical Gears With Curvilinear Shaped Teeth Cut by a Hob Cutter[J]. Journal of Mechanical Design, 2004, 127(5):271-277. https://doi.org/10.1115/esda2004-58176

[5] Dai Yutang, Ariga Yukinori, Jiang Desheng. Hobbing Mechanism of Cylindrical Gear with Arcuate Tooth Traces and Experimental Investigation [J]. China Mechanical Engineering, 2006, 17(7): 706-709.

[6] Wu Y C, Chen K Y, Tsay C B, et al. Contact Characteristics of Circular-Arc Curvilinear Tooth Gear Drives[J]. Journal of Mechanical Design, 2009, 131(8):81003-81010. https://doi.org/10.1115/1.3151805

[7] Ma Zhenqun, Deng Chengyi. CNC Machining Method of Whole Modified Surface of Cylindrical Gears with Arcuate Tooth Trace [J]. JOURNAL OF MECHANICAL ENGINEERING, 2012, 48(5):165-171. https://doi.org/10.3901/jme.2012.05.165

[8] Zhao Fei, Hou Li et al. Research on the Forming Theory Analysis and Digital Model of Circular Arc Gear Shaped by Rotary Cutter [J]. JOURNAL OF SICHUAN UNIVERSITY (ENGINEERING SCIENCE EDITION),2016,48(06):119-125.

[9] Andrei L, Andrei G. ANALYSIS OF GEAR QUALITY CRITERIA AND PERFORMANCE OF CURVED FACE WIDTH SPUR GEARS[C]// 2008.

[10] Fuentes A, Ruiz-Orzaez R, Gonzalez-Perez I. Computerized design, simulation of meshing, and finite element analysis of two types of geometry of curvilinear cylindrical gears[J]. Computer Methods in Applied Mechanics \& Engineering, 2014, 272(2):321-339. https://doi.org/10.1016/j.cma.2013.12.017

[11] Chen Y C, Lo C C. Contact stress and transmission errors under load of a modified curvilinear gear set based on finite element analysis[J]. ARCHIVE Proceedings of the Institution of Mechanical Engineers Part C Journal of Mechanical Engineering Science 1989-1996 (vols 203-210), 2015, 229(2):191-204. https://doi.org/10.1177/0954406214532907

[12] Sun Z, Hou L, Wang J, et al. Contact strength analysis of circular-arc-tooth-trace cylindrical gear[J]. Journal of the Brazilian Society of Mechanical Sciences \& Engineering, 2016, 38(3):999-1005. https://doi.org/10.1007/s40430-014-0272-6 
Cylindrical Gears

[13] Simon, Vilmos V . Machine-Tool Settings to Reduce the Sensitivity of Spiral Bevel Gears to Tooth Errors and Misalignments[J]. Journal of Mechanical Design, 2008, 130(8):082603. https://doi.org/10.1115/1.2936903

[14] Simon, Vilmos V . Optimization of face-hobbed hypoid gears[J]. Mechanism \& Machine Theory, 2014, 77:164-181. https://doi.org/10.1016/j.mechmachtheory.2014.02.003

[15] Tang Jinyuan, Lu Yanfeng,et al.Error Tooth Contact Analysis of Spiral Bevel Gears Transmission[J]. CHINESE JOURNAL OF MECHANICAL ENGINEERING,2008(07):16-23. https://doi.org/10.3901/jme.2008.07.016

[16] Chen Shuhan, YAN Hong-zhi, MING Xing-zu. Analysis and modeling of error of spiral bevel gear grinder based on multi-body system theory[J] J. Cent. South Univ. Technol. (2008) 15: 706-711 https://doi.org/10.1007/s11771-008-0131-7

[17] Ming Xingzu, Wang Wei, et al. Comprehensive Error Modeling and Analysis of Face Gear NC Grinding Machine Based on Multi-body System Theory [J] . Journal of Mechanical Transmission, 2015, 39 ( 4) : 32-36

[18] Zhou Chao,TANG Jinyuan, et al. Relationship between Grinding Wheel and Tooth Surface Error [J]. CHINESE JOURNAL OF MECHANICAL ENGINEERING,2008(02):94-101. https://doi.org/10.3901/jme.2008.02.094

[19] Wang Yanzhong, CHU Xiaomeng, et al. Impact of Machine Error on the Gear Tooth Surface Shape of Face Gear [J]. JOURNAL OF BEIJING UNIVERSITY OF TECHNOLOGY,2018,44(07):1017-1023.

[20] R.L.Houston, Liu Youwu, Dynamics of Multi-body System(Volume I)[M]. Tianjin:Tianjin University Press, 1987

[21] Chen Shuhan.Research on generation mechanism and correction technique for spiral bevel gear's grinding errors[D]. Central South University, 2009.

[22] Sristava A K, Veldhuis S C , Elbestawit M A . Modelling Geometric and Thermal Error in a Five-Axis CNC Machine Tool[J]. Int.j.mach.tools \& Manufac, 1995, 35(9):1321-1337.

https://doi.org/10.1016/0890-6955(94)00048-o

Submitted: $\quad 08.10 .2019$

Accepted: $\quad$ 16.5.2020
Yang $\mathrm{Wu}^{*}$

Li Hou

Luo Lan

Sichuan University, Chengdu, China

Dengqiu Ma

College of Eng. and Technol.,Zunyi

Normal College, Zunyi, China

Yongqiao Wei

School of Mechatronics Eng., Lanzhou

Univ. of Technol., Lanzhou, China

*walkerwuy@outlook.com 